\title{
Butyrate-Loaded Chitosan/Hyaluronan Nanoparticles: A Suitable Tool for Sustained Inhibition of ROS Release by Activated Neutrophils
}

Sacco, Pasquale; Decleva, Eva; Tentor, Fabio; Menegazzi, Renzo; Borgogna, Massimiliano; Paoletti, Sergio; Kristiansen, Kåre Andre; Vårum, Kjell Morten; Marsich, Eleonora

Published in:

Macromolecular Bioscience

Link to article, DOI:

10.1002/mabi.201700214

Publication date:

2017

Document Version

Peer reviewed version

Link back to DTU Orbit

Citation $(A P A)$ :

Sacco, P., Decleva, E., Tentor, F., Menegazzi, R., Borgogna, M., Paoletti, S., Kristiansen, K. A., Vårum, K. M., \& Marsich, E. (2017). Butyrate-Loaded Chitosan/Hyaluronan Nanoparticles: A Suitable Tool for Sustained Inhibition of ROS Release by Activated Neutrophils. Macromolecular Bioscience, 17(11), [1700214]. https://doi.org/10.1002/mabi.201700214

\section{General rights}

Copyright and moral rights for the publications made accessible in the public portal are retained by the authors and/or other copyright owners and it is a condition of accessing publications that users recognise and abide by the legal requirements associated with these rights.

- Users may download and print one copy of any publication from the public portal for the purpose of private study or research.

- You may not further distribute the material or use it for any profit-making activity or commercial gain

- You may freely distribute the URL identifying the publication in the public portal 


\section{Butyrate-Loaded Chitosan/Hyaluronan Nanoparticles: a Suitable Tool for Sustained Inhibition of ROS Release by Activated Neutrophils}

Pasquale Sacco ${ }^{*}$, Eva Decleva, Fabio Tentor, Renzo Menegazzi, Massimiliano Borgogna, Sergio Paoletti, Kåre Andre Kristiansen, Kjell Morten Vårum†, Eleonora Marsich.

P. Sacco*, E. Decleva, R. Menegazzi, M. Borgogna, Prof. S. Paoletti

Department of Life Sciences, University of Trieste

Via L. Giorgieri 5, I-34127 Trieste, Italy

*E-mail: psacco@units.it

F. Tentor

Department of Life Sciences, University of Trieste

Via L. Giorgieri 5, I-34127 Trieste, Italy

AND

Department of Micro- and Nanotechnology, Technical University of Denmark

Building 345C, 2800 Kgs. Lyngby, Denmark

K. A. Kristiansen, Prof. K. M. Vårum†

Norwegian Biopolymer Laboratory (NOBIPOL), Department of Biotechnology

Norwegian University of Science and Technology (NTNU), NO-7491 Trondheim, Norway

$\dagger$ Deceased on May 29, 2017

E. Marsich

Department of Medical, Surgical and Health Sciences, University of Trieste

Piazza dell'Ospitale 1, I-34129 Trieste, Italy 


\begin{abstract}
Tissue damage caused by excessive amounts of neutrophil-derived reactive oxygen species (ROS) occurs in many inflammatory diseases. Butyrate is a short-chain fatty acid (SCFA) with known antiinflammatory properties, able to modulate several neutrophil functions. Evidence is provided here that butyrate inhibits neutrophil ROS release in a dose- and time-dependent fashion. Given the short half-life of butyrate, chitosan/hyaluronan nanoparticles are next designed and developed as controlled release carriers able to provide cells with a long-lasting supply of this SCFA. Notably, while the inhibition of neutrophil ROS production by free butyrate declines over time, that of butyrate-loaded chitosan/hyaluronan nanoparticles (B-NPs) is sustained. Additional valuable features of these nanoparticles are inherent ROS scavenger activity, resistance to cell internalization and mucoadhesiveness. B-NPs appear as promising tools to limit ROS-dependent tissue injury during inflammation. Particularly, by virtue of their mucoadhesiveness, B-NPs administered by enema can be effective in the treatment of inflammatory bowel diseases.
\end{abstract}

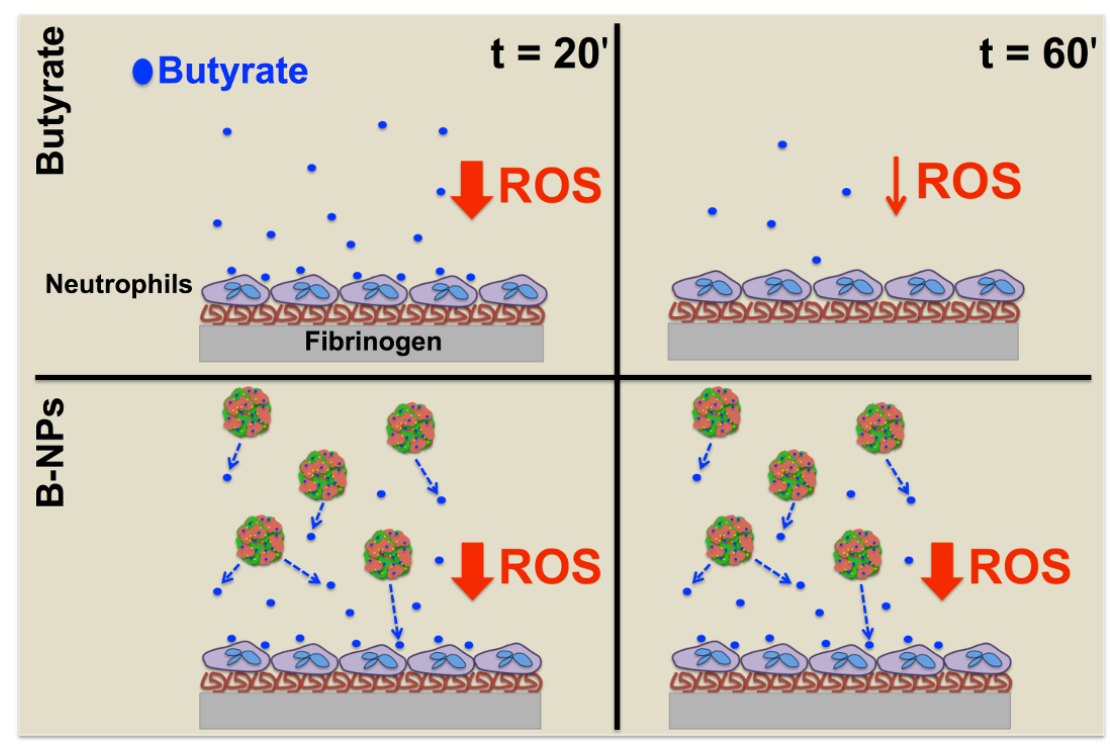




\section{Introduction}

The activation of neutrophil recruited at inflamed sites leads to the release of reactive oxygen species (ROS) and other pro-inflammatory mediators which, on one hand, contribute to neutrophil microbicidal activity and, on the other, are involved in the pathogenesis of tissue damage. Actually, tissue destruction and excessive inflammatory reaction contributed by neutrophil-derived toxic agents is known to occur in many inflammatory diseases. ${ }^{[1]}$ It is therefore comprehensible that, in an effort to hinder the "dark side" of inflammation, a huge amount of studies are continuously performed worldwide, with the aim of finding drugs that can eliminate, or at least attenuate, inflammation-derived tissue injury.

Short chain fatty acids (SCFAs), e.g. acetate, propionate and butyrate (herein termed NaBut), are the major metabolic products of the anaerobic fermentation of indigestible dietary fibers carried out by microbiota colonizing the large intestine. Besides being the preferred energy source for the cells that lay within the colon, SCFAs have been shown to possess other major biologic properties, including anti-inflammatory and anti-tumorigenic activities. ${ }^{[2],[3]}$ For instance, both in animal models and clinical trials, NaBut administration through oral route or enemas showed to be beneficial for the prevention and treatment of inflammatory bowel diseases. ${ }^{[4],[5]}$ The role of SCFAs in the modulation of the inflammatory response is mainly related to their effects on endothelial cells and leukocyte pathophysiology. Most of the studies deal with the capability of NaBut to influence several functional responses of neutrophils, which play a crucial role during the acute phase of the inflammatory reaction. It has been reported that NaBut modulates neutrophil adhesion, chemotaxis, degranulation, phagocytosis, microbial killing and the release of pro-inflammatory mediators. ${ }^{[6]}$ As far as ROS production is concerned, the effect of SCFAs, including butyrate, on neutrophil respiratory burst $(\mathrm{RB})$ remains a controversial issue. Indeed, both stimulatory ${ }^{[7],[8],[9]}$ and inhibitory activities ${ }^{[10],[11],[12],[13],[14]}$ on ROS release have been reported. Notably, the vast majority of these studies were performed exclusively on neutrophils maintained in suspension, a situation that is closer to the condition of circulating leukocytes than of activated cells in inflamed tissues. 
In the first part of our work, we revisited the effect of NaBut on human neutrophil RB by adopting an experimental model that more properly mimics the inflammatory environment, i.e. cells activated on biological substrates that support leukocyte adhesion (fibronectin: FN, fibrinogen: FBG). We show here that NaBut does not affect neutrophil adhesion, but it reduces the RB driven by both physiologically relevant stimuli (such as the proinflammatory cytokine tumor necrosis factor-alpha (TNF), or the bacterial chemotactic peptide N-formyl-L-methionyl-L-leucyl-Lphenylalanine, fMLP) and the non-physiological agonist, phorbol 12-myristate 13-acetate (PMA). Inhibition of ROS release is sustained within 10 - 30 min of cell stimulation with the agonists but appears to significantly decrease thereafter. Further observations reported in our study suggest that this decay may be reasonably ascribed to the rapid clearance of $\mathrm{NaBut}$ by human neutrophils. Indeed, previously published data have demonstrated the short half-life of butyrate both in vitro and in vivo. ${ }^{[15]}$

In order to extend over time the inhibitory effect of $\mathrm{NaBut}$ on neutrophil ROS production, we devised polysaccharide nanoparticles based on oppositely charged chitosan $(\mathrm{CH})$ and sodium hyaluronate (HA), capable of encapsulating and gradually releasing NaBut in the extracellular medium, thus providing cells with a continuous supply of the SCFA. Similarly to liposomes-like vesicles, ${ }^{[16],[17],[18]}$ micelles ${ }^{[19]}$ or more complex vectors, ${ }^{[20],[21]}$ chitosan-based nanoparticles are currently exploited as drug/gene delivery systems. ${ }^{[22],[23],[24]}$ The implementation of constructs with HA [25],[26],[27],[28],[29] has been shown to confer higher resistance to protein absorption, ${ }^{[30]}$ prolongation of nanoparticles half-life when circulating in the blood vessels, ${ }^{[31]}$ reduction of uptake by macrophages ${ }^{[32]}$ and the possibility to selectively target CD44 overexpressing cells. ${ }^{[33]}$

As a matter of fact, carriers for butyrate (namely: cholesteryl butyrate solid lipid nanoparticles) able to inhibit neutrophil superoxide anion $\left(\mathrm{O}_{2}{ }^{-}\right)$release more effectively than free NaBut have already been described. ${ }^{[34]}$ However, the results shown in that study refer to cells in suspension, which produce a limited amount of $\mathrm{O}_{2}{ }^{-}$upon activation, and the reported measurements have been performed over a short time interval (5 to $25 \mathrm{~min}$ ). Moreover, solid lipid nanoparticles 
are susceptible to rapid uptake by neutrophils, a feature conflicting with the need for a prolonged presence of NaBut in the extracellular compartment.

In this work we provide evidence that NaBut-loaded $\mathrm{CH} / \mathrm{HA}$ nanoparticles (B-NPs) sustain the inhibitory effect of $\mathrm{NaBut}$ on neutrophil ROS generation throughout the investigated time (60 min), thus preventing the decay observed when cells are exposed to free butyrate. This result, along with the fact that plain nanoparticles (NPs) exhibit ROS-scavenging activity and resistance to cell internalization, brings out the promising use of $\mathrm{CH} / \mathrm{HA}$ nanoparticles for delivering NaBut to sites of neutrophil accumulation in order to dampen ROS-dependent tissue injury. Moreover, in light of their mucoadhesive properties, we suggest that B-NPs-containing enemas could represent an applicable therapeutic intervention for patients suffering from inflammatory bowel diseases.

\section{Experimental Section}

\subsection{Materials}

Hydrochloride chitosans $(\mathrm{CH})$ were kindly provided by Prof. Kjell Morten Vårum (NTNU, Trondheim, Norway). The characteristics of $\mathrm{CH}$ are presented in Table S1. Sodium hyaluronate (HA), (viscosity average molecular weight, $\overline{M_{v}}=90000$, Bioibérica S.A.) was kindly provided by Sigea Srl (Trieste, Italy). Sodium tripolyphosphate pentabasic (TPP $\geq 98 \%$ ), NaBut (98\%), N-(3dimethylaminopropyl)-N'-ethylcarbodiimide hydrochloride (EDC), $N$-hydroxysuccinimide (NHS), O-benzylhydroxylamine (O-BHA), Percoll, Phosphate Buffered Saline (PBS), 2-(NMorpholino)ethanesulfonic acid (MES), bovine serum albumin (Cohn fraction V BSA, $\geq 96 \%$ cell culture-tested), cytochrome $c$ (type VI from horse heart), Ampliflu ${ }^{\mathrm{TM}}$ Red (AR) for fluorescence, $\geq$ 98.0\%, PMA, fMLP, xanthine, xanthine oxidase, from bovine milk, superoxide dismutase (SOD, from bovine erythrocytes), horseradish peroxidase (HRP), type VI, FBG, from human plasma, mucin (from porcine stomach, type III, bound sialic acid $0.5-1.5 \%$, partially purified) and pertussis toxin (PTX, from Bordetella pertussis) were all purchased from Sigma-Aldrich Co. (St. Louis, 
MO). FN, from human plasma, was from Roche Diagnostics GmbH (Penzberg, Germany). Human recombinant TNF- $\alpha$ (from yeast) was obtained from Miltenyi Biotec GmbH (Bergisch Gladbach, Germany). High-purity Trypan Blue (TB, Color Index 23850) - obtained from Merck KgaA (Darmstadt, Germany) - was dissolved in distilled water at $5 \mathrm{mg} \mathrm{mL}^{-1}$ and filtered through a Millipore filter to remove non-solubilized material. All reagents and chemicals were of high purity grade. All solutions used in the biological assays were prepared in endotoxin-free water or physiologic saline $(0.9 \% \mathrm{w} / \mathrm{v} \mathrm{NaCl})$ for clinical use.

\subsection{Preparation of Nanoparticles}

CH/HA-nanoparticles were fabricated according to the protocol reported elsewhere ${ }^{[25]}$ with slight modifications. $\mathrm{CH}\left(0.6 \mathrm{mg} \mathrm{mL}^{-1}\right)$ and $\mathrm{HA}\left(1.25 \mathrm{mg} \mathrm{mL}^{-1}\right)$ solutions were prepared by dissolving the polymers in deionized water. After complete solubilization, solutions were filtered $(0.22 \mu \mathrm{m}$ filters, Biosigma, Italy) and stored at room temperature until use. NPs were fabricated starting from the addition of $100 \mu \mathrm{L}$ of TPP $\left(0.5 \mathrm{mg} \mathrm{mL}^{-1}\right.$ in deionized water $)$ to $2 \mathrm{~mL}$ of HA under stirring condition. $0.5 \mathrm{~mL}$ of the resulting solution were then added dropwise to $0.5,1$ or $2 \mathrm{~mL}$ of $\mathrm{CH}$, in order to vary the HA: $\mathrm{CH}$ weight ratio. Formation of nanoparticles occurred within 10 min of incubation under stirring. For the preparation of B-NPs, NaBut was first solubilized in deionized water at different concentrations $(135,67.5,33.75,13.5$ and $1.35 \mathrm{mM}) .80,120$ and $200 \mu \mathrm{L}$ of each NaBut solution were then mixed with $0.5,1$ and $2 \mathrm{~mL}$ of $\mathrm{CH}$ solution, respectively, under stirring. The synthesis of B-NPs occurred in the same experimental conditions described above. The final NaBut concentration in B-NPs solutions was $10,5,2.5,1$ and $0.1 \mathrm{mM}$, respectively. The resulting mixtures were then held at room temperature for $30 \mathrm{~min}$ before use.

\subsection{Physicochemical Characterization of Nanoparticles}

\subsubsection{Nanoparticle Size, Surface Charge, and Morphology}

The size of both NPs and B-NPs was determined using Dynamic Light Scattering (DLS) on a 
Zetasizer Nano ZS with $173^{\circ}$ detection optics (Malvern Instruments). Measurements were performed in deionized water at $25^{\circ} \mathrm{C}$. All samples were diluted 1:10 v/v prior to analysis, to adjust scattering intensity. Nanoparticle size was expressed as Z-average hydrodynamic diameter, which was obtained by cumulative analysis of the correlation function, using the viscosity and refractive index of water in the calculations. $\zeta$-potential values were determined by means of laser Doppler velocimetry.

Images of NPs were acquired by Transmission Electron Microscopy (TEM). NPs were contrasted for $10 \mathrm{~min}$ using uranyl acetate $1 \% \mathrm{w} / \mathrm{v}$, diluted $1: 10 \mathrm{v} / \mathrm{v}$ in deionized water and gently deposited onto copper grids. Visual analysis and image recording were performed using a PHILIPS EM Transmission Electron Microscope.

\subsubsection{Stability Studies}

The stability of B-NPs was studied both in deionized water and in PBS buffer, by following the Zaverage hydrodynamic diameter variations upon dilution of the nanoparticles $(1: 10 \mathrm{v} / \mathrm{v})$ in either medium. The preparations were analyzed by DLS in the same experimental conditions reported above. When B-NPs were diluted in PBS, the final $\mathrm{pH}$ and ionic strength of the medium were 7.4 and $149 \mathrm{mM}$, respectively. Stability of the nanoparticles over time was monitored at $37^{\circ} \mathrm{C}$ up to three days, using B-NPs in deionized water. The stability of B-NPs was additionally evaluated by quantifying the amount of fluoresceinamine isomer I-labeled HA (see Supporting Information) released from nanoparticles after centrifugation $(12000 \times \mathrm{g}, 1 \mathrm{~h})$. Free-labeled polymer, at the same concentration as in the B-NPs, was also centrifuged and used as control (100\% of HA released). After centrifugation, supernatants were analyzed by a FLUOStar Omega-BMG Labtech spectrofluorometer $\left(\lambda_{\mathrm{ex}}=485 \mathrm{~nm} ; \lambda_{\mathrm{em}}=520 \mathrm{~nm}\right)$. The fluorescence intensity of each sample was expressed as percent of control fluorescence (see above). Data are reported as the difference between fluorescence intensities of supernatants recovered from B-NPs dispersed in PBS buffer ( $\left.\mathrm{I}_{\mathrm{PBS}}\right)$ and from B-NPs dispersed in deionized water $\left(\mathrm{I}_{\mathrm{DW}}\right)$. The results were interpreted as follows: 
$\mathrm{I}_{\mathrm{PBS}}-\mathrm{I}_{\mathrm{DW}}=0$ indicate stability; $\mathrm{I}_{\mathrm{PBS}}-\mathrm{I}_{\mathrm{DW}}>0$ indicate dissolution; $\mathrm{I}_{\mathrm{PBS}}-\mathrm{I}_{\mathrm{DW}}<0$ indicate aggregation.

\subsubsection{Encapsulation Efficiency}

To assess the encapsulation efficiency of NaBut-loaded nanoparticles, B-NPs were centrifuged $(3000 \times \mathrm{g}, 10 \mathrm{~min})^{[25]}$ and the un-encapsulated NaBut was measured in the supernatant. Encapsulation efficiency was calculated using the equation $[(\mathrm{A}-\mathrm{B}) / \mathrm{A}] \times 100$ where $\mathrm{A}$ and $\mathrm{B}$ are the concentrations of $\mathrm{NaBut}$ before and after the centrifugation, respectively. Supernatants were analyzed by mass spectrometry in ESI negative mode on a Waters XevoTQ-S instrument coupled to an I-Class UPLC in SIR (single ion recording) mode. The SIR ion was set to $\mathrm{m} / \mathrm{z}$ 87.1. UHPLC was run isocratically using $30 \%$ water $+0.025 \mathrm{mM} \mathrm{NH}_{3} \mathrm{OH}$ as mobile phase A and $70 \%$ acetonitrile $(\mathrm{ACN})+0.025 \mathrm{mM} \mathrm{NH}_{3} \mathrm{OH}$ as mobile phase $\mathrm{B}$ on a Waters BEH HILIC $130 \AA 1.7 \mu \mathrm{m}, 2.1 \mathrm{~mm} \mathrm{x}$ $100 \mathrm{~mm}$ column. A mixture of $10 \% \mathrm{ACN} / 90 \%$ water $+0.1 \%$ formic acid was used as wash and purge solvent. Flow rate was set to $0.150 \mathrm{~mL} \mathrm{~min}^{-1}$ and column temperature to $40{ }^{\circ} \mathrm{C}$. Injection volume was in the range $2-10 \mu \mathrm{L}$. Total run time was 2.5 min per sample. Capillary voltage was set to $2.5 \mathrm{kV}$, desolvation temperature $500{ }^{\circ} \mathrm{C}$, desolvation gas flow $1000 \mathrm{~L} \mathrm{hr}^{-1}$ and cone voltage 20 $\mathrm{kV}$. Dwell time was set to $0.15 \mathrm{~s}$ to get a minimum of 15 data points across the peak. Calibration curves $\left(R^{2}>0.99\right)$ were performed and run in parallel.

\subsection{In Vitro Release Studies}

The leakage of NaBut from B-NPs was measured in PBS buffer $(\mathrm{pH} 7.4$, final ionic strength $=166$ $\mathrm{mM})$ at $37^{\circ} \mathrm{C} .0 .1 \mathrm{~mL}$ of $10 \times$ PBS were added to $0.9 \mathrm{~mL}$ of B-NPs in deionized water $\left(681 \mu \mathrm{g} \mathrm{mL}^{-}\right.$

${ }^{1}$ final concentration). The resulting dispersions were sealed in a dialysis tube (Sigma-Aldrich, cutoff $12 \mathrm{kDa}$ ) and flooded in $2 \mathrm{~mL}$ of PBS under shaking conditions. The dialysis solution was collected and replaced with fresh PBS after each time investigated. The amount of NaBut in each dialysis sample was quantified by UHPLC-mass spectrometry in ESI positive mode, after 
derivatization with $O$-BHA and EDC (see Supporting Information). The release of NaBut from BNPs was monitored up to three days and results were reported as the percentage of cumulative release over time.

\subsection{Neutrophil Isolation}

Institutional ethics committee approval was obtained and written informed consent was signed by healthy volunteers from which venous blood was withdrawn. Neutrophils were isolated by a discontinuous Percoll gradient centrifugation, as described previously, ${ }^{[35]}$ and suspended in $\mathrm{Ca}^{2+}$ and $\mathrm{Mg}^{2+}$-free PBS solution, $\mathrm{pH} 7.4$, containing $5 \mathrm{mM}$ glucose and $0.2 \% \mathrm{w} / \mathrm{v}$ BSA (PBS-BSA).

\subsection{Preparation of FN- and FBG-Coated Surfaces}

Flat-bottom poly(styrene) wells (F16 MaxiSorp Nunc-Immuno ${ }^{\mathrm{TM}}$ Modules, ThermoFisher Scientific Inc., Waltham, MA) were coated with FN or FBG as described elsewhere. ${ }^{[36]}$ Briefly, $50 \mu \mathrm{L}$ of FN or FBG solution $\left(20 \mu \mathrm{g} \mathrm{mL} L^{-1}\right.$ and $400 \mu \mathrm{g} \mathrm{mL}^{-1}$ in PBS, respectively) were put in each well, and the plate was left at $37{ }^{\circ} \mathrm{C}$ for $1-2 \mathrm{~h}$ in a humidified chamber. Just before use, the wells were rinsed three times with PBS.

\subsection{Assay of $\mathrm{O}_{2}^{-}$Production}

$\mathrm{O}_{2}{ }^{-}$production was measured by the SOD-inhibitable cytochrome $c$ reduction assay, as previously described ${ }^{[37]}$ Briefly, freshly isolated neutrophils were suspended at $3.75 \times 10^{6}$ cells $\mathrm{mL}$ in PBSBSA and restored for $30 \mathrm{~min}$ at $37^{\circ} \mathrm{C}$ in a shaking water bath. Five to ten min before starting the assay, the cell suspension was supplemented with $1 \mathrm{mM}$ of $\mathrm{CaCl}_{2}$ and $1 \mathrm{mM}$ of $\mathrm{MgCl}_{2}$. Aliquots $(20 \mu \mathrm{L})$ were then added to FN- or FBG-coated wells containing cytochrome $c(0.12 \mathrm{mM}$ final concentration), NaBut (0.01 - $50 \mathrm{mM}$ final concentration), or nanoparticles $\left(480 \mu \mathrm{g} \mathrm{mL} \mathrm{L}^{-1}\right.$ final concentration) in a total volume of $0.16 \mathrm{~mL}$ PBS-BSA supplemented with $1 \mathrm{mM} \mathrm{CaCl}_{2}$ and $1 \mathrm{mM}$ $\mathrm{MgCl}_{2}\left(\mathrm{Ca}^{2+} / \mathrm{Mg}^{2+}\right.$ PBS-BSA). Immediately after the addition of the cells, $20 \mu \mathrm{L}$ of TNF $\left(10 \mathrm{ng} \mathrm{mL}^{-}\right.$ 
${ }^{1}$ final concentration), fMLP (5 $\mu \mathrm{M}$ final concentration) or PMA (20 ng mL ${ }^{-1}$ final concentration) were pipetted in each well. The plate was then transferred onto a thermostated microplate reader (MultiskanMCC/340; LabsystemOy, Helsinki, Finland) and readings were taken at intervals of 2.5 min for $60-90 \mathrm{~min}$. The amount of reduced cytochrome $c$ was calculated from the absorbance difference between $550 \mathrm{~nm}$ and $540 \mathrm{~nm}$, using an absorbance of 0.037 optical density (OD) units for 1 nmol reduced cytochrome $c$ as a standard.

\subsection{Evaluation of $\mathrm{O}_{2}^{-}$Scavenging Activity}

The superoxide radical scavenging effect of NPs was investigated using the xanthine-xanthine oxidase (XOX) system. $\mathrm{O}_{2}^{-}$generation by the XOX system was measured by a cytochrome $c$ reduction assay as previously described, with slight modifications. ${ }^{[38]}$ Xanthine $(0.05 \mathrm{mM}$ final concentration), cytochrome $c(0.02 \mathrm{mM}$ as final concentration) and, when needed, NPs, CH, HA or NaBut were added to microplate wells and the reaction was started by the addition of xanthine oxidase $\left(0.0025 \mathrm{U} \mathrm{mL}^{-1}\right.$ final concentration) to reach the final volume of $200 \mu \mathrm{L}$ in PBS. Final concentration of SOD, added as positive control, was $2.5 \mu \mathrm{g} \mathrm{mL}{ }^{-1}$. The plate was incubated at $37^{\circ} \mathrm{C}$ in a thermostated microplate reader and readings were taken at $550 \mathrm{~nm}$ and $540 \mathrm{~nm}$ at the selected times.

\subsection{Measurement of $\mathrm{H}_{2} \mathrm{O}_{2}$ Production}

Hydrogen peroxide production was measured using AR reagent. ${ }^{[39]}$ Neutrophils $\left(1 \times 10^{6}\right.$ cells $\mathrm{mL}$ in PBS-BSA) were pre-warmed for $30 \mathrm{~min}$ at $37{ }^{\circ} \mathrm{C}$ in a shaking water bath. Five minutes before starting the assay, the cell suspension was supplemented with $1 \mathrm{mM} \mathrm{CaCl} 2,1 \mathrm{mM} \mathrm{MgCl}, \mathrm{NaN}_{3}$ (100 $\mu \mathrm{M}$, final concentration) and $\operatorname{SOD}(5 \mu \mathrm{g} \mathrm{mL}-1$, final concentration). $75 \mu \mathrm{L} /$ well of the suspension were then dispensed into FBG-coated wells. After addition of $20 \mu \mathrm{L}$ of nanoparticles (80 - $250 \mu \mathrm{g} \mathrm{mL}^{-1}$ final concentration) or $\mathrm{NaBut}(0.5 \mathrm{mM}$ final concentration), the assay was started by dispensing $25 \mu \mathrm{L} /$ well of TNF (10 ng mL $\mathrm{m}^{-1}$, final concentration) in a total volume of $0.2 \mathrm{~mL}$ 
$\mathrm{Ca}^{2+} / \mathrm{Mg}^{2+}$ PBS-BSA. At every time point, $25 \mu \mathrm{L} /$ well of a solution containing $\mathrm{AR}$ (40 $\mu \mathrm{M}$ final concentration) and HRP (1 $\mu \mathrm{g} \mathrm{mL} \mathrm{m}^{-1}$ final concentration) (AR-HRP) were added to selected wells and the fluorescence associated to each well was read in a microplate fluorescence reader (Tecan Infinite F200; Tecan Austria GmbH, Grödig, Austria) at $535 \mathrm{~nm}\left(\lambda_{\mathrm{ex}}\right)$ and $595 \mathrm{~nm}\left(\lambda_{\mathrm{em}}\right)$.

\subsection{Measurement of Neutrophil Adhesion}

The number of neutrophils adherent to FN or FBG was assessed by quantifying myeloperoxidase activity as described in ${ }^{[35]}$, in the same wells in which $\mathrm{O}_{2}{ }^{-}$release had been measured. In the experiments aimed at assessing the $\mathrm{H}_{2} \mathrm{O}_{2}$ production, adhesion was evaluated in tests run in parallel without the final addition of AR-HRP, to avoid interference by HRP.

\subsection{Cellular Membrane Association and Internalization of Nanoparticles}

The association of fluorescence-labeled NPs with neutrophil membrane and their internalization were determined by means of flow cytometry according to the method described by Busetto et al., ${ }^{[40]}$ with slight modifications (see Supporting Information).

\subsection{Evaluation of Nanoparticle Mucoadhesiveness}

The mucoadhesive properties of NPs were evaluated in vitro by assessing the interaction between nanoparticles and mucin. Mucin was purified via dialysis against deionized water prior to use. The solution was thereafter filtered through $8 \mu \mathrm{m}$ Millipore filters and freeze-dried for storage. Freezedried mucin was solubilized in deionized water at $2 \mathrm{mg} \mathrm{mL}^{-1}$ prior to use. NPs $\left(80 \mu \mathrm{g} \mathrm{mL}^{-1}\right.$ final concentration in deionized water) were added $(1: 1 \mathrm{v} / \mathrm{v}, 1 \mathrm{~mL}$ final volume) to solutions of mucin at different concentrations $\left(0.2,1\right.$, and $\left.2 \mathrm{mg} \mathrm{mL}^{-1}\right)$. The resulting mixtures, that have a final $\mathrm{pH}$ of $\sim$ 5.5 , were vigorously resuspended and analyzed by DLS. ${ }^{[41]}$ The change in NPs size distribution was used as a parameter to evaluate aggregation phenomena.

\subsection{Statistical Analysis}


Statistical analysis was performed with Student's t test, using GraphPad Prism 5.0 (GraphPad Software, San Diego, CA). $P$ values less than 0.05 were considered statistically significant.

\section{Results and Discussion}

\subsection{Effect of Free NaBut on Neutrophil $\mathrm{O}_{2}^{-}$Production and Adhesion}

Neutrophil production of $\mathrm{O}_{2}^{-}$in response to an activating stimulus results from the assembly and activation at the plasma membrane of the enzymatic complex NADPH oxidase. The inflammatory cytokine TNF, one of the most important physiological trigger of NADPH oxidase activity, is able to induce a robust superoxide generation only in neutrophils adherent to extracellular matrix proteins that allow cell spreading. Consequently, we evaluated the effect of NaBut on the RB induced by TNF in neutrophils placed on FN. Figure 1A shows the dose-response curve of the effect of NaBut on TNF-triggered $\mathrm{O}_{2}{ }^{-}$release. Almost superimposable results were obtained with cells adherent to FBG (not shown), a biologic surface on which, as previously reported, ${ }^{[42]}$ neutrophils respond to TNF with a RB akin to that measured on FN. As described in the Experimental Section, neutrophils were always exposed to butyrate immediately before addition of the stimulus, since we noticed a significant decay of the inhibitory effect depending on the duration of the pretreatment with $\mathrm{NaBut}$ (Figure S1). At any NaBut concentration tested, $\mathrm{O}_{2}{ }^{-}$production by SCFA-treated cells was significantly lower than that of untreated cells. Moreover, tested NaBut concentrations that exerted inhibitory activity were quite lower than those generally reported in the literature. ${ }^{[10],[34],[43]}$ Such a discrepancy might be well justified by considering that (i) published results are obtained mostly with cells in suspension, that behave very differently from neutrophils in adherent conditions, and (ii) preincubation of cells with NaBut before addition of agonist is a common procedure ${ }^{[10],[34]}$ that could however limit butyrate efficacy (see our results reported in Figure S1), warranting the use of high concentrations to achieve a significant effect. In this regard, it is noteworthy that at concentrations equal or higher than $5 \mathrm{mM}$ (Table S2), the effect of butyrate 
was superimposable to that of equimolar solutions of $\mathrm{NaCl}$ or sodium gluconate. This implies that at high $\mathrm{NaBut}$ concentrations the observed inhibition of neutrophil RB is likely due to the increased tonicity of the medium rather than to a specific effect of the SCFA. In fact, it has been shown that the functional responses of adherent neutrophils are susceptible to even slight hypertonic conditions. $^{[44]}$

Since the metabolic activation of neutrophils in response to TNF is strictly adhesiondependent, ${ }^{[45]}$ we wondered if the inhibitory effect of NaBut on superoxide production might be due merely to an interference with cell attachment and/or spreading. The results we obtained prove that this was not the case, as the presence of NaBut did not affect neither adhesion (Figure 1B) nor cell morphology, as judged by optical microscopy (not shown).

Time course experiments were performed to better characterize the effectiveness of NaBut in reducing TNF-induced $\mathrm{O}_{2}^{-}$production. Figure $1 \mathrm{C}$ shows that, from 10 to $30 \mathrm{~min}$ of incubation, the inhibitory effect of $\mathrm{NaBut}$ ranged from nearly $55 \%$ to $47 \%$ and than tended to decline over time (nearly 35, 26, and 23\% at 40, 50 and $60 \mathrm{~min}$, respectively). The same time-dependence was also observed when fMLP and PMA were used to stimulate superoxide generation. In fact, with both agonists, the NaBut inhibitory effect was comparable to that seen with TNF, being strong (nearly $50 \%$ ) at earlier incubation times but declined at $60 \mathrm{~min}$ (Figure S2).

A possible explanation for this trend comes from the experiments performed with PTX and summarized in Figure 1D. As a G-protein inhibitor, PTX hinders the signaling pathway of the Gprotein coupled receptor 43 (GPR43), which recognizes butyrate and is highly expressed on neutrophils. ${ }^{[6],[7]}$ As shown in Figure 1D, the inhibitory effect of NaBut on TNF-induced superoxide production is abolished in the presence of PTX, indicating that it is mainly due to the interaction of NaBut with GPR43, as already demonstrated for butyrate-induced chemotaxis. ${ }^{[6],[46]}$ Since butyrate uptake by neutrophils is likely to be rapid, as proved for other cell types, ${ }^{[47]}$ we can hypothesize that the observed short-term inhibitory effect depends on the actual butyrate concentration in the incubation medium, which is expected to rapidly decrease with time thus limiting butyrate binding 
to GPR43. This is supported also by our preliminary observation on the drastic reduction of NaBut effect when neutrophils were pre-incubated with the SCFA prior to TNF exposure (Figure S1).

\subsection{Synthesis and Characterization of B-NPs}

Previously published data, and our own experimental observations, attesting the short half-life of butyrate in the extracellular environment, prompted us to design a carrier to be used as a reservoir for NaBut. With this aim, we synthesized NaBut-loaded biopolymer nanoparticles based on $\mathrm{CH}$ and HA in order to provide neutrophils with a long-lasting supply of the SCFA.

The synthesis of nanoparticles took place in a step-by-step process depicted in Figure 2A. Firstly, NaBut was mixed with $\mathrm{CH}$, a step during which weak electrostatic interactions between the carboxylic group of butyrate and the positive amino groups of $\mathrm{CH}$ are expected to occur. When HA/TPP was dropped into the $\mathrm{CH}-\mathrm{NaBut}$ mixture, the resulting solution became progressively turbid, an event indicative of nanoparticle formation. It is conceivable that the driving forces for nanoparticles self-assembling were the multivalent polyanion/polycation electrostatic interactions between the polymers, the ability of $\mathrm{CH}$ to undergo a liquid-gel transition in presence of TPP and, possibly, other interchain interactions (e.g., hydrogen bonds). ${ }^{[27]}$

Chitosans with different fractions of acetylated units $\left(\mathrm{F}_{\mathrm{A}}\right)$ were used, and the HA:CH weight ratio was varied, to fabricate a large number of formulations. DLS and $\zeta$-potential analyses were performed to investigate dimensions, surface charge and polydispersity of the nanoparticles. The results of this study are reported in Table S3. On varying the $\mathrm{F}_{\mathrm{A}}$ of the chitosan used, the formation of NPs with different characteristics was achieved: dimensions spanned from a little less than 200 $\mathrm{nm}$ to almost $800 \mathrm{~nm}$, whereas $\zeta$-potential ranged from $-25 \mathrm{mV}$ to about $+50 \mathrm{mV}$. TEM images of the nanocomposites in dried state (Figure 2B-C) confirmed the presence of nanoparticles with a round-shape morphology and with dimensions approximately similar to those calculated by means of DLS measurements.

By comparing the various formulations of NaBut-free nanoparticles, interesting features can 
be noticed. NPs made of chitosan with $\mathrm{F}_{\mathrm{A}}<0.008$ were found to gradually decrease dimensions as the HA:CH weight ratio was increased. It is expected that when an excess of $\mathrm{HA}$ is added to $\mathrm{CH}$, it is likely that the former tends to form a more cross-linked and therefore shrunken core due to high amine content of chitosan. ${ }^{[48]}$ Moreover, the increase of $\mathrm{HA}: \mathrm{CH}$ weight ratio affected the surface charge of the NPs, which switched from $\sim+52$ to $-25 \mathrm{mV}$ when the HA: $\mathrm{CH}$ mass ratio was varied from 1:2 to 2:1. An excess of $\mathrm{HA}$ with respect to $\mathrm{CH}$ is likely to form also a coating, covering the ionically cross-linked core, according to the core-shell model already proposed for either chitosan and alginate or chitosan and dextran complexes. ${ }^{[49],[50]}$ This HA surplus was found to positively narrow the size distribution of NPs, as demonstrated by the decrease of the polydispersity index (PDI) (Table S3). Indeed, as pointed out in Figure 2D, the NPs with a HA:CH weight ratio of 2:1 displayed a narrow distribution ranging from 90 to $500 \mathrm{~nm}$, with a maximum DLS intensity at about $200 \mathrm{~nm}$.

In the case of NPs made of chitosan with $\mathrm{F}_{\mathrm{A}}=0.64$, increasing the amount of HA did not significantly influence the dimension of the nanoparticles (from 196 to $230 \mathrm{~nm}$ when the HA:CH mass ratio was tuned from $1: 2$ to $2: 1$ ). This could be ascribed to the low availability, in the highly acetylated $\mathrm{CH}$, of positive amino groups able to interact with $\mathrm{HA}$, as proved by the lower $\zeta$ potential values of the nanoparticles. On the other hand, hydrophobic interactions throughout chitosan/hyaluronan chains could cooperate for the complex coacervation of polysaccharides. At variance with the NPs made of $\mathrm{CH}$ with $\mathrm{F}_{\mathrm{A}}<0.008$, the PDI progressively increased with increasing amounts of HA.

When NaBut was added to either chitosans and the formation of B-NPs took place, some differences were identified with respect to NPs (Table S3). The presence of NaBut within the nanoparticles affected their hydrodynamic diameter, surface charge and PDI. More in detail, the higher was the initial concentration of NaBut the lower was the $\zeta$-potential of all tested formulations, thus confirming the occurrence of electrostatic interactions between the SCFA and CH. Nevertheless, a simple physical entrapment cannot be excluded, which would successfully 
contribute to the encapsulation of $\mathrm{NaBut}$.

It has to be said that we performed some attempts to separate the nanoparticles from the reaction medium by means of centrifugation, a method reported in the majority of previously published articles describing similar systems. In our experimental conditions, however, centrifugation of the nanoparticles was not applicable since it caused an irreversible alteration of their microstructure: indeed, the visible pellet formed at the bottom of the microtube after centrifugation was not resuspendable anymore. Tirelli and co-workers reported the use of high molecular weight cutoff tubes $(1000 \mathrm{kDa})$ as an alternative method for separating nanoparticles from the reaction solution. ${ }^{[48]}$ Even if this approach can be considered the most suitable one for the isolation of $\mathrm{CH} / \mathrm{HA}$ nanoparticles, we did not utilize it because of the fast leakage of NaBut (see below), and consequent loss of payload during dialysis. Hence, both NPs and B-NPs were simply dispersed in different media after synthesis, without any intermediate step.

\subsection{Stability of B-NPs, Identification of the Best Formulation and Encapsulation Efficiency}

The colloidal stability of B-NPs is a pivotal feature for an efficient use of such nanosystems. Opposite charged polyelectrolyte complexes are strongly affected by the presence of salts (due to screening effects among charges) and $\mathrm{pH}$ variations. ${ }^{[51],[49]}$ For instance, it has been already demonstrated that the stability of $\mathrm{CH} / \mathrm{HA}$-based nanoparticles prepared in water is impaired upon dilution in PBS. ${ }^{[52]}$ This could represent the major issue for their potential use in both in vitro and in vivo models. Recently, $\mathrm{Wu}$ et al. demonstrated that $\mathrm{CH} / \mathrm{HA}$-polyelectrolyte complexes can be

stabilized in physiological conditions by the addition of $\mathrm{Zn}$ (II) ${ }^{[53]}$ but biocompatibility analyses, that are mandatory for any in vitro/in vivo application of such a system, are not available yet.

In the present work we evaluated the stability of B-NPs upon dilution either in deionized water or in PBS (Figure 3). B-NPs made of chitosan with $\mathrm{F}_{\mathrm{A}}<0.008$ and HA:CH of 1:2 were greatly affected by dilution in PBS: nanoparticles rapidly increased their dimensions until reaching 
roughly $1.8 \mu \mathrm{m}$ as average hydrodynamic diameter (Figure 3A). Aggregation was confirmed by visible formation of flocculated suspensions after $24 \mathrm{~h}$ of incubation.

In an attempt to get insights into the mechanisms governing nanoparticle aggregation, we performed some experiments in which the ionic strength and $\mathrm{pH}$ of the dispersion medium were alternately modulated. We observed that, when $\mathrm{NaCl}$ was added to B-NPs dispersed in deionized water to reach a $0.9 \% \mathrm{w} / \mathrm{v}$ final concentration (corresponding to $\mathrm{I}=154 \mathrm{mM}$ ), the particle size distribution increased in parallel. On the contrary, no aggregation occurred when the $\mathrm{pH}$ of the dispersion was tuned within the range 7.0 - 7.4 (and I $\sim 0$ ) (data not shown). Hence, the reason for nanoparticle aggregation in PBS is most likely the ionic strength of the medium. By increasing the amount of HA, the aggregation (and the consequent flocculation) decreased, as shown in Figure 3A: at a $\mathrm{HA}: \mathrm{CH}$ ratio of 2:1 the resulting B-NPs dispersed in PBS were only slightly larger than those in deionized water. As shown in Figure 3C, the latters retained their dimension up to three days of incubation at $37^{\circ} \mathrm{C}$.

On the other hand, the $\mathrm{Z}$-average size of $\mathrm{B}$-NPs made of $\mathrm{CH}$ with $\mathrm{F}_{\mathrm{A}}=0.64$ and dispersed in either water or PBS was almost comparable (Figure 3B). More in detail, a slight aggregation, without flocculation, occurred in PBS using formulations with HA:CH weight ratio of 1:1 and 2:1, whereas for nanoparticles with $\mathrm{HA}: \mathrm{CH}$ weight ratio of 1:2 a reduction in Z-average was noticed. A possible explanation for such a different behavior, with respect to the nanoparticles made of chitosan with $\mathrm{F}_{\mathrm{A}}<0.008$, is that the high amount of acetylated units of $\mathrm{CH}\left(\mathrm{F}_{\mathrm{A}}=0.64\right)$ ensures its solubility at physiological $\mathrm{pH}$ thus preventing precipitation and, at the same time, lowers the impact of ionic strength on the electrostatic interchain interactions. As a consequence, screening effects do not dramatically trigger aggregation and the interchain forces between the polysaccharides mostly ensure the retention of dimensions. As observed for $\mathrm{F}_{\mathrm{A}}<0.008$ chitosan $\mathrm{B}-\mathrm{NPs}, \mathrm{F}_{\mathrm{A}}=0.64$ chitosan B-NPs were found to be stable in deionized water at $37{ }^{\circ} \mathrm{C}$, since they showed only a slight increment in dimensions at $48 \mathrm{~h}$ of incubation, probably due to swelling phenomena (Figure 3C). 
Generally, dilution in PBS was accompanied by an increased polydispersity of all formulations (PDI >0.35), which is likely accounted for by the rearrangement of the polysaccharide chains (with consequent broadening of the size distribution). To further elucidate how this impacted on the final structure of B-NPs, the stability of nanoparticles in deionized water and in PBS was also studied by assessing the amount of fluorescence-labeled HA released from B-NPs after centrifugation. Stable formulations are expected to exhibit in the supernatants the same fluorescence intensity after centrifugation in either deionized water or PBS. Conversely, a change in fluorescence after dilution and centrifugation of B-NPs in PBS would be indicative of nanoparticle dissolution (higher fluorescence intensity) or aggregation (lower intensity). Figure 3D points out that all B-NPs were affected by the dilution in PBS. As regards nanoparticles with $\mathrm{F}_{\mathrm{A}}<0.008$ chitosan, only in the case of HA:CH 1:2 the fluorescence measured in PBS was lower than that in deionized water, thus suggesting the early formation of macroaggregates which conceivably sank to the bottom of the tubes during centrifugation; this result confirmed the data obtained by DLS (see Figure 3A). For the other weight ratios analyzed, a partial dissolution of B-NPs was observed, as the fluorescence in the PBS supernatant was higher than that in deionized water. It can be inferred that, albeit aggregation occurred as demonstrated by DLS analysis, the dispersion of nanoparticles in PBS caused also a partial release of HA from B-NPs. With regard to the nanoparticles built with $\mathrm{F}_{\mathrm{A}}=0.64$ chitosan, the dilution in PBS dramatically affected the stability of the formulation with $\mathrm{HA}: \mathrm{CH}=1: 2$, since after centrifugation HA was almost completely recovered in the supernatant. This explains the reduction of the Z-average size observed by DLS measurements (Figure 3B), which can be therefore ascribed to a substantial dissolution of B-NPs. By increasing the ratio between HA and $\mathrm{CH}$, the dissolution of B-NPs was clearly limited (Figure 3D). Overall, one can conclude that, although the dispersion (dilution) of B-NPs in PBS seems to induce most of B-NPs to aggregate, a partial loss of polysaccharides from the nanoparticles, caused by slight dissolution, cannot be excluded. 
Considering all the analyzed features (particle size, polydispersity index, surface charge and stability; see Table S3 and Figure 3), and the evidence that NaBut has a prominent inhibitory effect on neutrophil $\mathrm{O}_{2}^{-}$production at a concentration of $1 \mathrm{mM}$ (Figure 1A), the best candidate formulation for further studies was considered to be

Chitosan with $\mathrm{F}_{\mathrm{A}}=0.64$; NaBut $1 \mathrm{mM}$; HA:CH 1:1

The encapsulation efficiency of NaBut for these B-NPs, as calculated by means of UHPLCmass spectrometry, was $70.0 \pm 1.4 \%$.

\subsection{Release of NaBut from B-NPs}

Before assaying the effect of B-NPs on neutrophil RB, time-course experiments were run to evaluate the release of NaBut from the nanoparticles in PBS buffer. As shown in Figure 4, the leakage profile of NaBut from B-NPs was almost constant in the first $20 \mathrm{~min}$, with approximately $45 \%$ of the SCFA being released from the nanoparticles. For longer incubation times, the leakage tended to slow down and to progressively reach a plateau. The cumulative release reached $\sim 87 \%$ after $4 \mathrm{~h}$ of incubation. It is noteworthy to mention that such a release did not significantly vary up to $72 \mathrm{~h}$ (not shown) and never reached $100 \%$, possibly because a small amount of NaBut remained entrapped in, and permanently contributed to the B-NP architecture. Overall, these results indicate that $\mathrm{CH} / \mathrm{HA}$ nanoparticles behave as a restraint to NaBut diffusion (free NaBut diffused more than $90 \%$ from dialysis bag after $1 \mathrm{~h}$ of incubation under the same experimental conditions, data not shown). B-NPs appear therefore suitable for achieving a controlled release of the SCFA during the early stages of the inflammatory reaction, when neutrophil-mediated ROS production needs to be restrained.

\subsection{Effect of NPs and B-NPs on ROS Production by TNF-Stimulated Neutrophils}

When NPs and B-NPs of the selected formulation were tested on neutrophils, NPs displayed an inhibitory effect on $\mathrm{O}_{2}^{-}$which was superimposable to that of B-NPs, as pointed out in Figure 5A. 
Since HA and $\mathrm{CH}$ are well known to behave as radical scavengers, ${ }^{[54]}$ we assumed that the observed effect did not rely on the inhibition of NADPH oxidase activity but, rather, on the scavenging of superoxide by the nanoparticle components. Figure 5B shows, indeed, that addition of NPs to the xanthine-xanthine oxidase $(\mathrm{XOX})$ system, which generates $\mathrm{O}_{2}^{-}$, results in about $40 \%$ decrease in cytochrome $c$ reduction, while separate addition of $\mathrm{HA}$ or $\mathrm{CH}$, at the concentration at which they are present in the NPs, leads to a decrease of about $20 \%$. This reveals that NPs are endowed per se with a significant $\mathrm{O}_{2}^{-}$scavenging activity (inset to Figure 5B), which corresponds approximately to the sum of the individual effects of each polysaccharide component. On one hand, this finding opens an encouraging perspective, since delivering NaBut by a radical scavenger carrier would enhance the anti-inflammatory potential of the formulation. On the other hand, the scavenging activity of NPs hinders the correct determination of the effect of B-NPs on NADPH oxidase activity in the $\mathrm{O}_{2}^{-}$production assay.

To circumvent this methodological drawback, we next assessed the effect of B-NPs on the RB by measuring $\mathrm{H}_{2} \mathrm{O}_{2}$ production in the presence of SOD, which rapidly converts all extracellular $\mathrm{O}_{2}^{-}$ into $\mathrm{H}_{2} \mathrm{O}_{2}$. $\mathrm{NaN}_{3}$ was also added to the incubation medium to prevent $\mathrm{H}_{2} \mathrm{O}_{2}$ degradation by endogenous catalase. ${ }^{[55]}$ Figure 6 shows that, in these experimental conditions, NPs still retained a minor inhibitory effect, which decreased with time and could be reasonably attributable to the $\mathrm{H}_{2} \mathrm{O}_{2}$ scavenging ability of both $\mathrm{HA}$ and $\mathrm{CH}^{\left[{ }^{[54]}\right.}$ B-NPs loaded with $1 \mathrm{mM}$ NaBut (Figure 6: (a) B-NPs) exerted an effect superimposable to that of NPs, possibly because of the low concentration of $\mathrm{NaBut}$ available to neutrophils over time (the final total concentration of NaBut in the wells was 0.1 $\mathrm{mM}$ ). In the light of these results, the possibility to increase the NaBut loading of B-NPs was considered. Concentrations of NaBut equal or higher than $10 \mathrm{mM}$ were not taken into account because of the too high PDI of the resulting B-NPs (Table S3). Hence, the inhibitory effect of tenfold dilutions of B-NPs loaded with 2.5 and $5 \mathrm{mM} \mathrm{NaBut}$ on neutrophil respiratory burst was tested (Figure 6, labels b and c, respectively). At $20 \mathrm{~min}$ of incubation, the most effective nanoparticles were the c B-NPs, which inhibited TNF-induced $\mathrm{H}_{2} \mathrm{O}_{2}$ release in a manner comparable to free 
NaBut. Moreover, it is worth noting that c B-NPs were able to sustain efficiently the inhibitory effect of NaBut throughout the assay: the percentage of inhibition induced by these nanoparticles was not significantly reduced from 20 to $60 \mathrm{~min}$, whereas the activity of NaBut decreased by $45 \%$, as similarly observed while measuring $\mathrm{O}_{2}{ }^{-}$production (Figure 1C). Since B-NPs loaded with $5 \mathrm{mM} \mathrm{NaBut}$ were almost comparable to those enriched with $1 \mathrm{mM}$ in terms of polydispersity and surface charge $(0.20 \pm 0.05$ and $-26 \pm 2 \mathrm{mV}$, respectively), we can consider this formulation as the most promising for forthcoming works.

\subsection{Cell Association/Internalization and Mucoadhesive Properties of NPs}

The association of fluorescence-labeled NPs with TNF-activated neutrophils, and nanoparticle cellular uptake, were investigated by means of cytofluorometric analysis (Figure 7). By comparing the percentage of neutrophils with associated NPs (upper left quadrants) of the first five panels, we can infer that adhesion of NPs to the cell surface was immediate (at time 0, red-fluorescent neutrophils represented already $\sim 34 \%$ of gated events) and did not increase significantly with time, remaining nearly constant throughout the $180 \mathrm{~min}$ incubation. The same panels also show that internalization of NPs by neutrophils was occasional, since green-fluorescent cell subsets in the upper plus lower right quadrants were less than $1 \%$ at any incubation time. This should suggest a receptor-mediated association, inasmuch the binding (and consequent uptake) of HA-coated nanoparticles by macrophage membrane receptors (e.g. CD44) has been shown. ${ }^{[4]}$ Contextually, the presence of HA seems being a factor necessary but not sufficient to obtain - and explain receptor CD44-mediated targeting, since the binding has been found to be rapid (in line with our results with human neutrophils), whereas internalization can be slow. ${ }^{[56]}$ In any case, NPsneutrophil association appeared to be weak: when cells were washed after incubation with NPs, an almost complete loss of membrane-associated nanoparticles was observed (Figure 7, sixth panel: Washed). 
Confocal microscopy observations confirmed the sporadic nature of NPs uptake by activated neutrophils (not shown). Since the inhibitory effect of NaBut on neutrophil ROS production seems exerted from the outside of the cell mainly via GPR43 receptors (see Figure 1D), the low internalization rate of B-NPs has to be considered a key feature, capable of conferring them a high advantage in comparison with carriers that undergo rapid cellular uptake. In fact, if the internalization of NaBut-loaded nanoparticles would occur too rapidly, the release of butyrate in the extracellular environment would be impaired, thus limiting the interaction with its membrane receptors and, ultimately, the efficacy of the treatment.

Taking into account the potential application of enema-administered B-NPs in the treatment of bowel inflammatory lesions, their mucoadhesive properties were also studied. Mucoadhesiveness was assessed by evaluating the interaction between the nanoparticles and mucin. ${ }^{[57]}$ Specifically, upon mixing NPs with mucin, variation of NPs size distribution was monitored by means of DLS measurements, and used as a parameter to quantify aggregation. While NPs alone usually showed a typical unimodal size distribution with low PDI values, mucin $\left(0.1 \mathrm{mg} \mathrm{mL}^{-1}\right)$ exhibited a nearly bimodal distribution (not shown), with the presence of two main peaks at $875 \pm 26$ and $92 \pm 13 \mathrm{~nm}$. This distribution closely resembles that reported by Sogias and coworkers, which demonstrated a similar bimodal curve for mucin at the concentration of $1 \mathrm{mg} \mathrm{mL}^{-1} \cdot{ }^{[41]}$ When NPs and mucin were mixed, increasing the amount of mucin led to a progressive increase of NPs distribution peak, thereby suggesting aggregation phenomena (Figure 8). Simultaneously, PDI values tended to gradually increase, thus confirming the onset of more variable colloidal aggregates formation. Sogias and co-workers concluded their work claiming that electrostatic interactions, hydrogen bonding and hydrophobic effects cooperate to favor the interactions between chitosan and mucin. With regard to the NPs used in our experiments, it is unlikely that electrostatic interactions may account for their mucoadhesiveness, as suggested by the negative $\zeta$-potential of the nanoparticles $(\sim$ $-20 \mathrm{mV})$ as well as the negative charge of mucin at the $\mathrm{pH}$ investigated $(\sim-18 \mathrm{mV}) \cdot{ }^{[41]}$ Conversely, both hydrogen bonding and hydrophobic effects might be considered at the root of their adhesive 
interactions with mucin.

\section{Conclusions}

In the first part of the present paper, we shed new light on the effect of free butyrate on ROS production by human neutrophils. Namely, we provided evidence that (i) NaBut is able to dampen the respiratory burst of activated, adherent neutrophils in a dose- and time-dependent manner; (ii) this effect is independent of the agonist used to trigger the RB, and (iii) the GPR43 receptor signaling pathway is involved in NaBut-induced restraint of neutrophil ROS release. The inhibitory activity of the SCFA was found to be sustained within 10 - 30 min of incubation and to decline afterwards. To avoid this decay, likely attributable to a drop in extracellular NaBut concentration due to rapid cellular uptake, we next addressed the design of suitable reservoirs for NaBut. CH/HAbased nanoparticles loaded with $\mathrm{NaBut}$ were fabricated and characterized for their physicochemical properties, stability, encapsulation efficiency and NaBut release. The results of these studies enabled us to identify the most promising formulation (B-NPs made of $\mathrm{CH}$ with $\mathrm{F}_{\mathrm{A}}=0.64$ and a $\mathrm{HA}: \mathrm{CH}$ weight ratio of $1: 1)$ that was subsequently tested on neutrophils. We show here that the selected B-NPs, loaded with $5 \mathrm{mM} \mathrm{NaBut}$, inhibited $\mathrm{H}_{2} \mathrm{O}_{2}$ release by neutrophils exposed to TNF, without displaying the significant decline of the inhibitory effect observed with free NaBut. Furthermore, we demonstrate that the $\mathrm{CH} / \mathrm{HA}$ nanoparticles are endowed with three valuable features that, to our knowledge, have not been described for other proposed carriers for NaBut: (1) inherent ROS scavenging ability, that would be additive to the inhibitory effect of NaBut on ROS release; (2) resistance to cell internalization and gradual NaBut release, that would prolong the presence of the SCFA in the extracellular microenvironment, thus ensuring an effective interaction with the transmembrane receptor GPR43, and (3) mucoadhesiveness, that would be favorable in prospect of a possible administration of B-NPs to protect inflamed mucous epithelia from ROSmediated damage. Particularly, the delivery of mucoadhesive B-NPs by means of enemas could be considered a potential therapeutic approach for patients suffering from inflammatory bowel 
diseases. In this regard, our in vitro studies lay the groundwork for further investigations on suitable animal models.

The overall results presented in this paper suggest that B-NPs, better than free NaBut, may be beneficial in the treatment of inflammatory conditions characterized by ROS-mediated tissue damage. Given the inherent properties of NPs uncovered here, we propose $\mathrm{CH} / \mathrm{HA}$ nanoparticles as promising vehicles also for delivering anti-inflammatory drugs other than butyrate.

Acknowledgments: The financial support to P.S. (Ph.D. scholarship) by the Friuli-Venezia Giulia Regional Government, by the European Social Fund (S.H.A.R.M. project-Supporting human assets in research and mobility) and by COST Action Number CM1101 is gratefully acknowledged.

Author contributions: Supplied materials: KMV, MB. Conceived and designed the experiments: PS, ED, FT, RM, SP, KMV, EM. Performed the experiments: PS, ED, FT, RM, KAK. Analyzed the data: PS, ED, FT, RM, MB, KMV, EM, KAK. Wrote the paper: PS, ED, FT, RM.

Keywords: chitosan/hyaluronan nanoparticles, butyrate, human neutrophils, reactive oxygen species, inflammation

\section{References}

[1] T. N. Mayadas, X. Cullere, C. A. Lowell, Annu. Rev. Pathol. 2014, 9, 181.

[2] P. Louis, G. L. Hold, H. J. Flint, Nat. Rev. Microbiol. 2014, 12, 661.

[3] J. Tan, C. McKenzie, M. Potamitis, A. N. Thorburn, C. R. Mackay, L. Macia, Adv. Immunol. 2014, $121,91$.

[4] L. Krokowicz, Z. Stojcev, B. F. Kaczmarek, W. Kociemba, E. Kaczmarek, J. Walkowiak, P. Krokowicz, M. Drews, T. Banasiewicz, Int. J. Colorectal Dis. 2014, $29,387$.

[5] R. G. Pacheco, C. C. Esposito, L. C. M. Müller, M. T. L. Castelo-Branco, L. P. Quintella, V. L. A. Chagas, H. S. P. de Souza, A. Schanaider, World J. Gastroenterol. 2012, 18, 4278.

[6] M. A. R. Vinolo, H. G. Rodrigues, R. T. Nachbar, R. Curi, Nutrients 2011, 3, 858.

[7] K. M. Maslowski, A. T. Vieira, A. Ng, J. Kranich, F. Sierro, D. Yu, H. C. Schilter, M. S. Rolph, F. Mackay, D. Artis, R. J. Xavier, M. M. Teixeira, C. R. Mackay, Nature 2009, 461, 1282. 
[9] S. Nakao, Y. Moriya, S. Furuyama, R. Niederman, H. Sugiya, Cell Biol. Int. 1998, 22, 331.

[10] M. A. R. Vinolo, E. Hatanaka, R. H. Lambertucci, P. Newsholme, R. Curi, Cell Biochem. Funct. 2009, $27,48$.

[11] A. Sandoval, F. Triviños, A. Sanhueza, D. Carretta, M. A. Hidalgo, J. L. Hancke, R. A. Burgos, Vet. Immunol. Immunopathol. 2007, 115, 286.

[12] C. Eftimiadi, E. Buzzi, M. Tonetti, P. Buffa, D. Buffa, M. T. J. van Steenbergen, J. de Graaff, G. A. Botta, J. Infect. 1987, 14, 43 .

[13] M. Tonetti, A. Cavallero, G. A. Botta, R. Niederman, C. Eftimiadi, J. Leukoc. Biol. 1991, 49, 180.

[14] Q. Liu, T. Shimoyama, K. Suzuki, T. Umeda, S. Nakaji, K. Sugawara, Scand. J. Gastroenterol. $2001,36,744$.

[15] L. Serpe, R. Canaparo, M. Daperno, R. Sostegni, G. Martinasso, E. Muntoni, L. Ippolito, N. Vivenza, A. Pera, M. Eandi, M. R. Gasco, G. P. Zara, Eur. J. Pharm. Sci. 2010, 39, 428.

[16] M. Ripoli, R. Angelico, P. Sacco, A. Ceglie, A. Mangia, J. Biomed. Nanotechnol. 2016, 12, 770.

[17] R. Angelico, A. Ceglie, P. Sacco, G. Colafemmina, M. Ripoli, A. Mangia, Int. J. Pharm. 2014, 471, 173.

[18] R. Molinaro, C. Corbo, J. O. Martinez, F. Taraballi, M. Evangelopoulos, S. Minardi, I. K. Yazdi, P. Zhao, E. De Rosa, M. B. Sherman, A. De Vita, N. E. Toledano Furman, X. Wang, A. Parodi, E. Tasciotti, Nat. Mater. 2016, $15,1037$.

[19] V. P. Torchilin, J. Control. Release 2001, 73, 137.

[20] A. Parodi, N. Quattrocchi, A. L. van de Ven, C. Chiappini, M. Evangelopoulos, J. O. Martinez, B. S. Brown, S. Z. Khaled, I. K. Yazdi, M. V. Enzo, L. Isenhart, M. Ferrari, E. Tasciotti, Nat. Nanotechnol. 2013, 8, 61.

[21] R. Xu, G. Zhang, J. Mai, X. Deng, V. Segura-Ibarra, S. Wu, J. Shen, H. Liu, Z. Hu, L. Chen, Y. Huang, E. Koay, Y. Huang, J. Liu, J. E. Ensor, E. Blanco, X. Liu, M. Ferrari, H. Shen, Nat. Biotechnol. 2016, $34,414$.

[22] J. Malmo, A. Sandvig, K. M. Vårum, S. P. Strand, PLoS One 2013, 8, e54182.

[23] G. Q. Teixeira, C. Leite Pereira, F. Castro, J. R. Ferreira, M. Gomez-Lazaro, P. Aguiar, M. A. Barbosa, C. Neidlinger-Wilke, R. M. Goncalves, Acta Biomater. 2016, 42, 168.

[24] K. A. Janes, M. P. Fresneau, A. Marazuela, A. Fabra, M. J. Alonso, J. Control. Release 2001, $73,255$.

[25] X. Deng, M. Cao, J. Zhang, K. Hu, Z. Yin, Z. Zhou, X. Xiao, Y. Yang, W. Sheng, Y. Wu, Y. Zeng, Biomaterials 2014, 35, 4333.

[26] M. de la Fuente, B. Seijo, M. J. Alonso, Nanotechnology 2008, 19, 075105.

[27] M. de la Fuente, B. Seijo, M. J. Alonso, Invest. Ophthalmol. Vis. Sci. 2008, 49, 2016.

[28] A. Almalik, R. Donno, C. J. Cadman, F. Cellesi, P. J. Day, N. Tirelli, J. Control. Release 2013, $172,1142$.

[29] A. Gennari, M. Pelliccia, R. Donno, I. Kimber, N. Tirelli, Adv. Healthc. Mater. 2016, 5, 966.

[30] T. I. Croll, A. J. O’Connor, G. W. Stevens, J. J. Cooper-White, Biomacromolecules 2006, 7, 1610.

[31] D. Peer, R. Margalit, Int. J. Cancer 2004, 108, 780.

[32] N. M. Zaki, A. Nasti, N. Tirelli, Macromol. Biosci. 2011, 11, 1747.

[33] S. Ouasti, P. J. Kingham, G. Terenghi, N. Tirelli, Biomaterials 2012, 33, 1120.

[34] C. Dianzani, R. Cavalli, G. P. Zara, M. Gallicchio, G. Lombardi, M. R. Gasco, P. Panzanelli, R. Fantozzi, Br. J. 
Pharmacol. 2006, 148, 648.

[35] R. Menegazzi, S. Busetto, P. Dri, R. Cramer, P. Patriarca, J. Cell Biol. 1996, 135, 511.

[36] P. Dri, R. Cramer, P. Spessotto, M. Romano, P. Patriarca, J. Immunol. 1991, 147, 613.

[37] R. Menegazzi, R. Cramer, P. Patriarca, P. Scheurich, P. Dri, Blood 1994, 84, 287.

[38] J. M. McCord, I. Fridovich, J. Biol. Chem. 1969, 244, 6049.

[39] J. G. Mohanty, J. S. Jaffe, E. S. Schulman, D. G. Raible, J. Immunol. Methods 1997, 202, 133.

[40] S. Busetto, E. Trevisan, P. Patriarca, R. Menegazzi, Cytometry. A 2004, 58, 201.

[41] I. A. Sogias, A. C. Williams, V. V Khutoryanskiy, Biomacromolecules 2008, 9, 1837.

[42] P. Dri, R. Cramer, M. Romano, P. Spessotto, P. Patriarca, Int. J. Tissue React. 1991, 13, 193.

[43] M. A. R. Vinolo, H. G. Rodrigues, E. Hatanaka, C. B. Hebeda, S. H. P. Farsky, R. Curi, Clin. Sci. (Lond). 2009, 117, 331 .

[44] B. A. Kruskal, S. Shak, F. R. Maxfield, Proc. Natl. Acad. Sci. U. S. A. 1986, 83, 2919.

[45] C. Nathan, S. Srimal, C. Farber, E. Sanchez, L. Kabbash, A. Asch, J. Gailit, S. D. Wright, J. Cell Biol. 1989, 109, 1341.

[46] L. B. Bindels, E. M. Dewulf, N. M. Delzenne, Trends Pharmacol. Sci. 2013, 34, 226.

[47] C. Hadjiagapiou, L. Schmidt, P. K. Dudeja, T. J. Layden, K. Ramaswamy, Am. J. Physiol. Gastrointest. Liver Physiol. 2000, 279, G775.

[48] A. Almalik, S. Karimi, S. Ouasti, R. Donno, C. Wandrey, P. J. Day, N. Tirelli, Biomaterials 2013, $34,5369$.

[49] H. V. Sæther, H. K. Holme, G. Maurstad, O. Smidsrød, B. T. Stokke, Carbohydr. Polym. 2008, 74, 813.

[50] C. Schatz, A. Domard, C. Viton, C. Pichot, T. Delair, Biomacromolecules 2004, 5, 1882.

[51] H. Dautzenberg, J. Kriz, Langmuir 2003, 19, 5204.

[52] Y. Parajó, I. D’Angelo, A. Welle, M. Garcia-Fuentes, M. J. Alonso, Drug Deliv. 2010, 17, 596.

[53] D. Wu, T. Delair, Carbohydr. Polym. 2015, 119, 149.

[54] S. Yang, Z. Guo, F. Miao, Q. Xue, S. Qin, Carbohydr. Polym. 2010, 82, 1043.

[55] E. Decleva, R. Menegazzi, A. Fasolo, F. Defendi, M. Sebastianutto, P. Dri, Exp. Cell Res. 2013, $319,1875$.

[56] J. M. Rios de la Rosa, A. Tirella, A. Gennari, I. J. Stratford, N. Tirelli, Adv. Healthc. Mater. $2017,6,1601012$.

[57] L. Yin, J. Ding, C. He, L. Cui, C. Tang, C. Yin, Biomaterials 2009, 30, 5691. 